\title{
DOES UNIVERSALISM ENTAIL EXTENSIONALISM?
}

\author{
A. J. COTNOIR \\ University of St Andrews
}

\begin{abstract}
Does a commitment to mereological universalism automatically bring along a commitment to the controversial doctrine of mereological extensionalism - the view that objects with the same proper parts are identical? A recent argument suggests the answer is 'yes'. This paper attempts a systematic response to the argument, considering nearly every available line of reply. It argues that only one approach — the mutual parts view — can yield a viable mereology where universalism does not entail extensionalism.
\end{abstract}

Here is an axiomatization of classical extensional mereology, where ' $<$ ' stands for the proper parthood relation, ' $\leq$ ' stands for the parthood relation (i.e. ' $x \leq y$ ' abbreviates ' $x<y \vee x=y$ '), and $\circ$ for mereological overlap (i.e. ' $x \circ y$ ' abbreviates ' $\exists z(z \leq x \wedge z \leq y$ )').

Transitivity $(x<y \wedge y<z) \rightarrow x<z$

Weak Supplementation $x<y \rightarrow \exists z(z \leq y \wedge \neg z \circ x))$

Unrestricted Fusion $\forall x x \exists y \mathrm{~F}(y, x x)$.

Where fusions are defined via:

Fusion $\mathrm{F}(t, x x):=x x \leq t \wedge \forall y(y \leq t \rightarrow y \circ x x))$

${ }^{*}$ Forthcoming in Nôेs. 
To simplify notation, I use ' $x x \leq y$ ' to mean that each $x$ among $x x$ is part of $y$; likewise ' $y \circ x x$ ' means that $y$ overlaps some $x$ among $x x$. It is worth noting that weak supplementation entails the remaining (strict) partial order axioms: ${ }^{1}$

Irreflexivity $x \nless x$

Asymmetry $x<y \rightarrow y \nless x$

The definition of $\leq$ provides the corresponding weak partial order principles of reflexivity and antisymmetry. Listing these as separate axioms is unnecessary here; however, this will not be so in what follows.

There is an argument (due to Achille Varzi [3 I]) based on this axiomatization that accepting Universalism (as expressed by the unrestricted fusion axiom) entails the acceptance of the controversial principle of extensionality. Extensionality states that composed objects with the same proper parts are identical. It is often thought that the first two axioms are unassailable (even analytic), and hence extensionality seems to rest only on the supposition of unrestricted fusion axiom — universalism entails extensionalism.

This entailment is extremely important, for extensionality is a philosophically contentious doctrine. It states, roughly, that any two composed objects with the same proper parts are identical. In other words, if objects are mereologically indiscernible, then they are indiscernible tout court. One might have thought that the debates regarding extensionality are largely independent of universalism. (Indeed, since the anti-extensionalist thinks objects with the same parts can be distinct, anti-extensionalism should be prima facie compatible with more fusions, not less!) But if the entailment is correct, it rules out any anti-extensionalist approach that wishes to countenance as many fusions as the classical mereologist. This has further consequences for other important metaphysical debates as well. For example, in the debate between endurantism and perdurantism over persistence, the argument rules out the viability of any form of plenitudinous coincidentalism

\footnotetext{
${ }^{1}$ For the first, suppose $x<x$. By weak supplementation, there must be part of $x$ that doesn't overlap $x$, which is impossible. For the second, suppose $x<y$ and $y<x$. Weak supplementation requires a part of $y$ disjoint from $x$. But by transitivity every part of $y$ is part of $x$, and hence every part of $y$ overlaps $x$. Contradiction.
} 
— a three-dimensionalist view of persistence (held by e.g. Hawthorne [I 4] and Bennett [I]) that allows for as many objects as a four-dimensionalist would countenance. Since the entailment has potentially widespread ramifications, we should care about determining whether it is true.

A streamlined presentation of Varzi's argument can be given as follows. Imagine you had a counterexample to extensionality; a statue $s$ and its clay $c$, suppose, are distinct composite objects with the same proper parts. By asymmetry, $s \nless c$, and $c \nless s^{2}{ }^{2}$ By unrestricted fusion, there must be a sum $s+c$ (which is distinct from either $s$ or $c$, since neither is part of the other). Now, $s<s+c$; however, there is no part of $s+c$ that is disjoint from $s$, as any part of $c$ is also part of $s$ by supposition. This violates weak supplementation. Hence, there can be no such counterexample to extensionality.

In this paper, I attempt to defend a version of universalism without extensionality against this argument. In $\S_{I}$, I consider a response to the argument regarding the definition of fusion (and correct an error of Varzi's along the way), showing this will not ultimately be successful. In $\$ 2$, I consider responses that drop supplementation principles altogether, and argue that these responses fail due the two important roles of supplementation in mereology. In $\S_{3}$, I outline and defend a version of universalist anti-extensionalism that avoids the argument and has serious advantages over its unsupplemented cousin. This view is what I'll call the mutual parts approach. I conclude that the mutual parts approach is the only anti-extensionalist view that survives the argument, and hence is the best version of universalist anti-extensionalism.

\section{Fussing with Fusions?}

Here is one possible line response to the entailment argument: one might try to appeal to an alternative definition of fusion to save universalism. Varzi considers this line of response and looks at each of $\mathrm{F}^{\prime}$-type and $\mathrm{F}^{\prime \prime}$-type universalism and argues that they are inadequate notions of fusion in the non-extensional context. (Of course, Varzi himself accepts them all, since in the

\footnotetext{
${ }^{2}$ For, suppose $c<s$. Asymmetry implies $s \nless c$. Hence, $s$ and $c$ are not a counterexample to extensionality. Mutatis mutandis for the supposition that $s<c$.
} 
extensional context they are equivalent.) ${ }^{3}$

Fusion' $\left.F^{\prime}(t, x x):=\forall y(y \circ t \leftrightarrow y \circ x x)\right)$

Fusion" $F^{\prime \prime}(t, x x):=x x \leq t \wedge \forall y(x x \leq y \rightarrow t \leq y)$

For the purposes of this essay, I concur with Varzi's arguments against $\mathrm{F}^{\prime}$-type fusions. ${ }^{4}$

Varzi's arguments against $F^{\prime \prime}$-type fusions, however, are incorrect. Varzi's main case against $\mathrm{F}^{\prime \prime}$-type fusions concerns the model below, where the domain is $\left\{x, y, z_{1}, z_{2}, z_{3}, w\right\}$ with proper parthood indicated by paths via upward arrows.

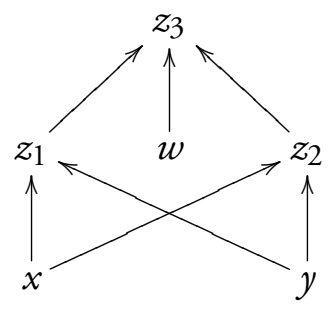

Varzi claims,

Now $z_{3}$ counts as an $\mathrm{F}^{\prime \prime}$-type fusion of $z_{1}$ and $z_{2}$, and inspection shows that every non-empty collection of elements has a fusion in this sense - a minimal upper bound. That's the good news for the universalist non-extensionalist, since the model is still in violation of [extensionality].

The difficulty here, is that the above model is simply not a model of the mereology under consideration. In particular, Varzi's claim that "every non-empty collection of elements has a fusion in this sense" is false. Not every non-empty collection of elements has an $F^{\prime \prime}$-type fusion.

Consider the collection containing just $x$ and $y$. We need to find some $\mathrm{F}^{\prime \prime}$-type fusion, namely some $t$ such that $x \leq t$ and $y \leq t$ and $\forall z((x \leq z \wedge y \leq z) \rightarrow t \leq z)$. Well, let us check. Is $z_{1}$

${ }^{3}$ Hovda [I 5] calls F-type fusion 'Type-2', F'-type fusions 'Type-I', and F''-type fusions 'Mub'. These also correspond to the definitions of fusion in Varzi [28], where he calls their corresponding existence axioms 'Strong Sumb', 'Strong Sum', and 'Strong Sum $a$ ' respectively. To gain CEM from $F^{\prime}$-type fusions, we need SSP (See \$4); with F'"type fusions, we need the even stronger SSSP (also in $\left.\$_{4}\right)$.

${ }^{4}$ However, once one drops antisymmetry, stronger supplementation principles can be put forward which make $F^{\prime}$-type fusions much more plausible. See [8] for details. 
such a $t$, is it an $\mathrm{F}^{\prime \prime}$-type fusion? No, because $x \leq z_{2}$ and $y \leq z_{2}$ but $z_{1} \not \leq z_{2}$. Is $z_{2}$ such a $t$ ? No, because $x \leq z_{1}$ and $y \leq z_{1}$ but $z_{2} \not \leq z_{1}$. Is $z_{3}$ an $\mathrm{F}^{\prime \prime}$-type fusion? No, because $x \leq z_{1}$ and $y \leq z_{1}$ but $z_{3} \not \leq z_{1}$. Obviously, $w$ can't be an $F^{\prime \prime}$-type fusion because it fails to have either $x$ or $y$ as parts. So, there is no $\mathrm{F}^{\prime \prime}$-type fusion of $x$ and $y$ in the model.

Dropping $x$ and $y$ from the model solves the issue, but then the model is no longer a countermodel to extensionality. So Varzi's reasons for rejecting $\mathrm{F}^{\prime \prime}$-type fusions are incorrect. However, there are better reasons for thinking that $F^{\prime \prime}$-type universalism is no good for the antiextensionalist. Here is a quite general result: if parthood is a partial order, then any model in which $F^{\prime \prime}$-type fusion is unrestricted simply cannot have a counterexample to extensionality. Why? Because in the presence of the partial order axioms, particularly antisymmetry, $F^{\prime \prime}$-type fusions guarantees the existence of a least upper bound. These must be unique. ${ }^{5}$ But counterexamples to extensionality require that for some parts $x x$, there are at least two distinct minimal upper bounds, and that none of them is least.

In fact, this general result has an important corollary. Another axiomatization of classical mereology consists in transitivity, weak supplementation, unrestricted $F^{\prime \prime}$-type fusions, plus the following principle:

Filtration $\forall y \forall z\left(\mathrm{~F}^{\prime \prime}(z, x x) \wedge y \leq z\right) \rightarrow y \circ x x$

Filtration is a straightforward principle that displays precisely how much weaker the $\mathrm{F}^{\prime \prime}$-type definition of fusion is than F-type definition of fusions. It is easy to see via some unabbreviation that in the presence filtration, $\mathrm{F}^{\prime \prime}$-type fusions just are $\mathrm{F}$-type fusions. ${ }^{6}$ In consequence, one can see from the general result above that dropping filtration from the above axiom set won't help the anti-extensionalist.

It appears Varzi had in mind a fourth type of fusion - minimal upper bounds - which generalises the least upper bounds of $\mathrm{F}^{\prime \prime}$-type fusions. ${ }^{7}$

\footnotetext{
${ }^{5}$ Suppose $\mathrm{F}^{\prime \prime}(t, x x)$ and $\mathrm{F}^{\prime \prime}(\hat{t}, x x)$. So, $x x \leq t$ and $x x \leq \hat{t}$ and $\forall y(x x \leq y \rightarrow t \leq y)$ and $\forall y(x x \leq y \rightarrow \hat{t} \leq y)$. It follows that $t \leq \hat{t}$ and $\hat{t} \leq t$. By antisymmetry $t=\hat{t}$.

${ }^{6}$ Hovda identifies this axiom; see $\left[\mathrm{I}_{5}\right] \S_{3}$.

${ }^{7}$ Indeed, Varzi has confirmed in correspondence that this is the notion he was intending.
} 
Fusion* $\mathrm{F}^{*}(t, x x):=x x \leq t \wedge \forall y(x x \leq y \rightarrow y \nless t)$

These are minimal upper bounds in the more usual mathematicians' sense for which the partial order axioms do not guarantee uniqueness. Consider again the above model; here is Varzi's criticism of it.

Something is clearly amiss here. For $z_{3}$ is not just composed of $z_{1}$ and $z_{2}$; it is also composed of $w$, which is disjoint from both. In fact, $z_{3}$ also counts as an $\mathrm{F}^{\prime \prime}$-type fusion of the two atoms $x$ and $w$ (leaving out $y$ ) and of the two atoms $y$ and $w$ (leaving out $x$ ). All this is hard to swallow. No matter how exactly one defines the word, surely a fusion is supposed to be entirely composed of the things it fuses.

So, Varzi's basic argument is that the notion of fusion in play makes very little sense given this model. The fusion of $x$ and $w$ contains more than just $x$ and $w-$ it contains something disjoint from either. But that cannot be an adequate notion of fusion, according to Varzi. ${ }^{8}$

Substituting ' $F *$-type fusion' for ' $F$ "-type fusion' in the Varzi's argument makes sense of the passage. That's because in the above model, $z_{1}$ and $z_{2}$ do count as $\mathrm{F}^{*}$-type fusions of $x$ and $y$; moreover everything in the model does have a fusion*. Indeed, all of his arguments against it go through mutatis mutandis. The problem with the model is that the fusion* of $z_{1}$ and $z_{2}$ contains more than it should - it contains $w$ which is disjoint from either. Can the anti-extensionalist rule models like this out?

Well, the model is a clear violation of the filtration principle for fusion*. Let $x x$ be $z_{1}$ and $z_{2}$. So, $\mathrm{F}^{*}\left(z_{3}, x x\right)$. Moreover, $w \leq z_{3}$ but it's simply not the case that $w \circ x x$. So a natural thought might be that adding filtration* should eliminate any problem of this sort. But, unfortunately, this rules out non-extensional models entirely!

\footnotetext{
${ }^{8}$ One obvious response is that perhaps sometimes fusions do contain more than just the parts contained in the things being fused. Consider for example, a philosopher who thought that boundary points are not real entities capable of independent existence. For her, the fusion of the open intervals $(0,1)$ and $(1,2)$ would be $(0,2)$. But the fusion then would contain a point that was not part of either. (Indeed, see Forrest [1o] for a mereology of this sort.) Is it plausible that such a view is conceptually impossible? Since this issue is independent of extensionalism, I'll set aside this type of response in what follows.
} 
Why? Well, let $s$ and $t$ be $\mathrm{F}^{*}$-type fusions of $x x$. Then by the definition of $\mathrm{F}^{*}, s \nless t$ and $t \nless s$. So, by unrestricted fusion*, there must be a fusion of $s$ and $t$ distinct from either. Call it $s+t$. Since $s<s+t$, by weak supplementation there must be some $w \leq s+t$ and disjoint from $s$. Now, if $w<t$ but not $s$, we no longer have a non-extensional model. And $w \neq t$ since $t$ is not disjoint from $s$. But if $w \nless t$ we violate filtration.

I conjecture there is no plausible strengthening of $\mathrm{F}^{*}$-type fusions to rule out unintended models whilst keeping anti-extensional ones. I am not aware of any other notions of fusion in the literature; if there are any, chances are good that they will face problems similar to the ones given above. It appears that fusions aren't to blame.

\section{Suspending Supplementation?}

It appears that the only remaining available responses to the above arguments are: (i) reject weak supplementation, or (ii) reject transitivity. I won't consider further option (ii). ${ }^{9}$ However, there is a growing number of mereologists who reject supplementation principles in mereology. ${ }^{10}$ Now, Varzi (and others) contend that supplementation is simply analytic; it is constitutive of the notion of proper parthood. ${ }^{11}$ Claims to analyticity of this sort are fairly flimsy, in my view, and we would do better to avoid relying on them.

Supplementation principles play a number of important roles in axiomatizations of mereology. Those roles are crucial in ruling out unintended models — indeed, often models that have nothing to do with failures of extensionality. I will focus here are two such roles: first, supplementation rules out the 'empty object'; second supplementation rules certain types of proper parthood chains which I'll call 'total for the model'. I think these roles can be used to argue that supplementation of some sort is necessary, on pain of accepting awkward models that do not directly contribute to the anti-extensionalist's cause. (As noted above, another role played by supplementation in Varzi's

\footnotetext{
${ }^{9}$ For the the purposes of the paper, I'll rely on the arguments in Varzi [29].

${ }^{10}$ Proponents include: [9, I O, 23, 24]. Also see Caplan et. al. [4] who express sympathy for the view.

${ }^{11}$ Varzi says this in several places: [30] (p. I Io) and [3 I] (p. 60). Simons [23] (p. I I6) also makes this claim, as do Bohn [2] (p. 27, footnote 3), Koslicki [18] (p. I67f), and McDaniel [20] (p. 264).
} 
axiomatization is entailing the partial order axioms. Proponents of unsupplemented mereology will want to reinstate reflexivity and antisymmetry as axioms.)

Here are some variant of supplementation principles that have been proposed.

Weak Supplementation (WSP) $x<y \rightarrow \exists z(z \leq y \wedge \neg z \circ x))$

Strong Supplementation (SSP) $y \not \leq x \rightarrow \exists z(z \leq y \wedge \neg z \circ x))$

Super-Strong Supplementation (SSSP) $y \not \leq x \rightarrow \exists z \forall w(w \leq z \leftrightarrow(w \leq y \wedge \neg w \circ x))^{12}$

In the presence of the partial order axioms, WSP, SSP, and SSSP are of increasing strength; that is, SSSP implies SSP, which implies WSP. ${ }^{13}$

An important role of supplementation principles is to rule out the 'empty' or 'null' object an object $x$ that is part of everything. (Of course, this isn't meant to exclude a one-element model — the model corresponding to 'existence monism'. The thought is that, so long as there is more than one object, there is no object that is part of everything.) An empty object has almost always been excluded from mereology, on the grounds that it is highly counterintuitive. ${ }^{14}$ So long as there are two objects, WSP, SSP, and SSSP rule out models with a bottom element. To see why, suppose there is an empty object 0 , such that 0 is part of everything, and another object $1 \neq 0$. Then $0<1$ satisfying the antecedent of WSP. But then by WSP, there must be some $z$ that is part of 1 but disjoint from 0 . But nothing is disjoint from 0 , by hypothesis. Contradiction.

As accepting the existence of an empty object is totally irrelevant to anti-extensionalism, there would seem to be good reason to search for an alternative to simply dropping WSP.

\footnotetext{
${ }^{12}$ Better known as 'Complementation' in the literature, also called the 'Remainder Principle' by Simons [23].

${ }^{13}$ For SSP entailing WSP: assume SSP; so in every case where $y \not \leq x$ there is an $x$-disjoint part of $y$. Now assume (for conditional proof) that $x<y$. By asymmetry, $y \nless x$, and since $x \neq y$, we have it that $y \nless \leq x$. Thus, there is an $x$-disjoint part of $y$. For SSSP entailing SSP, we just need to show that $\exists z \forall w(w \leq z \leftrightarrow(w \leq y \wedge \neg w \circ x))$ implies $\exists \hat{z}(\hat{z} \leq y \wedge \neg \hat{z} \circ x))$. But obviously, since by reflexivity $z \leq z$, we have it that $z \leq y \neg z \circ x$. Hence any witness for $z$ in SSSP is a witness for $\hat{z}$ of SSP.

${ }^{14}$ The lone exceptions are Bunt [3], Carnap [5], and Martin [19]. Calling it a 'fiction,' Simons calls advocates of it "culprit $[s]$ in propounding this absurdity" ([23, p. I3]). Geach [II, p. 522] responds to Carnap's proposal as absurd, by exclaiming that "the null thing is described as corresponding 'to the null class of space-time points' — or, in plain English, as existing nowhen and nowhere!"
} 
Gilmore [I 2] has recently endorsed dropping WSP and proposed the following principle as a substitute for it.

Quasi-Supplementation (QSP) $x<y \rightarrow \exists w \exists z(w \leq y \wedge z \leq y \wedge \neg z \circ w))$

Gilmore suggests that QSP gives formal expression to the informal intuitive thought that if an object has a proper part, then it must have two disjoint proper parts. This, he thinks, is the core thought driving the claims to WSP's analyticity. In addition to being weak enough to avoid Varzi's argument to extensionality, QSP also rules out the empty object. Assume that there are two objects 0 and 1 as before. Since $0<1$, by QSP, there must be some $w$ and $z$ that are both parts of 1, but don't overlap each other. But since 0 is part of everything, it must be part of both $z$ and $w$. Hence, they overlap. Contradiction.

However, there is an important role WSP, SSP, and SSSP play that is not fulfilled by QSP. WSP rules out the existence of certain kinds of chains - chains which are total for the model. More specifically, suppose we have a model $M$. We want to ask: are there linearly ordered subsets $S$ of $M$ such that there are no elements in $M$ incomparable with any element of $S$ ? WSP legislates the answer 'no' (at least if the subsets aren't mere singletons). QSP says 'yes'. In fact, there are models that satisfy QSP but with long chains that are total for the model. For example, consider the model below: 


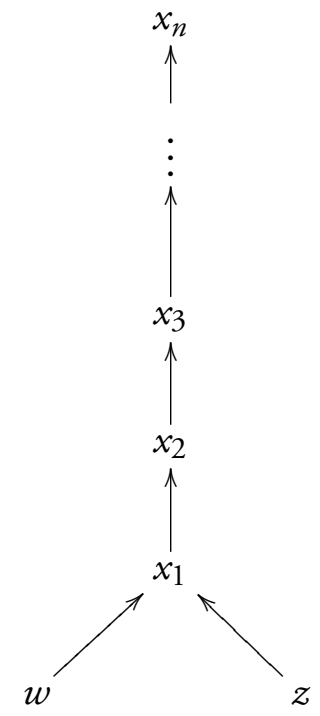

This model satisfies QSP, since $w$ and $z$ are disjoint parts of every $x_{i}$. QSP only forces composed objects to have disjoint proper parts somewhere down the line. But $x_{1}<\ldots<x_{n}$ is a totally ordered subset of the model, with no other elements of the model incomparable with any $x_{i}$. These structures seem much more akin to set theory, in which there is a clear difference between e.g. $\{\{\{w, z\}\}\}$ and $\{\{w, z\}\}$. They are less mereologically sound, and they clearly don't seem to map on to the intuitive gloss that 'an object that has a proper part must have two disjoint proper parts'. In any case, strange models of this sort have nothing to do with failures of extensionality. ${ }^{1516}$

\footnotetext{
${ }^{15}$ Gilmore [12] considers a model like the above model which contains $x_{2}$ and all its parts. Gilmore claims of the model:

[It] approximates the following view, which I regard as a respectable thing for a Coincidentalist to endorse: the statue and the lump are not identical, they both fuse the same set of simple particles, and the lump is part of the statue, but the statue is not part of the lump.
}

While this might be a respectable view for the coincidentalist, it is not a respectable view for the anti-extensionalist. After all, the model is not a counterexample to the extensionality of proper parthood. Moreover, this does not answer for the admissibility of the full range of mostly-linear models that QSP allows. I remain unconvinced that they have anything to do with anti-extensionalism.

${ }^{16}$ These models might, however, impinge on set-nominalism and the historical motivations of mereology. A typically nominalistic slogan is that there can be 'no difference without a difference maker'. Witness Goodman,

The platonist may distinguish these entities by venturing into a new dimension of Pure Form, but the nominalist recognizes no distinction of entities without a distinction of content. ([I3], 26)

So there may be a sense in which some further commitment to nominalism lobbies against both chains like these and non-extensional models. But simply because the anti-extensionalist accepts one counter instance to the nominalist slogan doesn't affect whether she should accept every such counterinstance. 
As such, I conclude that QSP is not an adequate anti-extensionalist substitute for WSP. What to do? There seems to be no coherent non-extensional mereology which accepts universalism.

\section{The Mutual Parts Approach}

There is another approach to anti-extensional mereology, which postulates the existence of distinct but mutual parts. On this approach, counterexamples to extensionality ought to be thought of as parts of each other. The view is due originally to Thomson [25, 26] (but see also Cotnoir [6] for a recent defence). It allows for models like the following, where $a$ and $b$ are taken to be coincident objects (e.g. the statue and the clay).

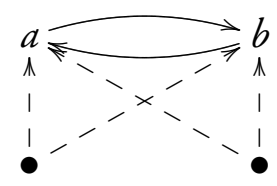

Of course, such models are incompatible with the antisymmetry of $\leq$, since that would imply mutual parts are identical. But above we showed that the asymmetry of $<$ (likewise, the antisymmetry of $\leq$ ) a theorem - it follows from weak supplementation. So the mutual parts theorist will not accept weak supplementation, as it is incompatible with her view. Moreover, antisymmetry has come under recent fire, independently of the extensionality debate. ${ }^{17}$

It may come as a surprise that once antisymmetry is rejected, supplementation principles like SSP or SSSP may be adopted with all their attendant benefits. As mentioned above SSP (and SSSP) entails WSP; but the proof relies crucially on antisymmetry. Moreover, in the absence

\footnotetext{
${ }^{17}$ There are a few main reasons for dropping antisymmetry. As above, co-location of material objects and the putative counterexamples to extensionality are one such reason (see Thompson [26] and Cotnoir [6]). Thomson thinks that the statue is constituted by the clay, and that constitution requires mutual parthood: ' $x$ constitutes $y$ at $t$ only if $x$ is part of $y$ at $t$ and $y$ is part of $x$ at $t^{\prime}$ (I 55). Constitution, Thomson thinks, does not imply identity; hence she rejects the antisymmetry axiom. Cotnoir [7] also notes that the mutual parts of co-location view allows one to preserve a principle of mereological harmony: if the location of $x$ is a subregion of the location of $y$, then $x$ is part of $y$.

But there are a number of extensionality-independent arguments, too. The first involves multiple location across time (see again Thomson [25]). The second involves multiple location across space (see Kleinschmidt [I7]). The third involves the apparent conceivability of putative examples of proper parthood loops (many of which are summarised in Cotnoir and Bacon [8]). See also Sanford [22] and Tillman and Fowler [27] for cases involving the universe being contained in some proper part of itself. For a concise introductory discussion of these issues, see Cotnoir [7].
} 
of antisymmetry, one cannot derive any extensionality principles. If composed objects have all the same parts, then all that follows is that they are mutual parts. Classically, one then uses antisymmetry to prove their identity. However, in this context, that move is not valid.

Significantly, these stronger supplementation principles make fusions behave nicely (even in the absence of antisymmetry). Take for example, the $F^{\prime \prime}$-type fusion axiom. When SSSP is present, this implies filtration. ${ }^{18}$ So none of our earlier issues with fusion arise.

Moreover SSP (and SSSP) satisfy a number of the roles that WSP typically plays. For one, it rules out the empty object. In the absence of antisymmetry, however, we need to be a bit careful how we define the empty object. For example, consider the following model in which the only objects present are mutual parts. Then each object counts as 'empty' given the old definition.

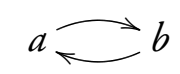

But clearly, these aren't the sort of models meant to be ruled out. An anti-extensionalist might wish to accept co-located simples (e.g. entangled quarks), which are not nearly as problematic as a null thing. No, the intended notion of an empty object corresponds to an object which is part of everything but which itself has no proper parts (i.e. $\forall y(0 \leq y \wedge y \nless 0)){ }^{19}$

Now suppose there exists an empty object (of the aforementioned sort) 0 , and another object $1 \neq 0$. Then $1 \not \leq 0$ satisfies the antecedent of SSP. But then by SSP, there must be some $z$ that is part of 1 but disjoint from 0 . But nothing is disjoint from 0 , by hypothesis. Contradiction.

Second, SSP (and SSSP) also rule out proper parthood chains of the questionable sort that QSP allows. For suppose that there are two objects, $x_{1}$ and $x_{2}$ such that $x_{1}<x_{2}$ but $x_{2} \nless x_{1}$.

\footnotetext{
${ }^{18}$ Suppose $\mathrm{F}^{\prime \prime}(a, x x)$. So $x x \leq a$. To prove filtration, suppose for reductio that $z \leq a$ and none $x x$ overlaps $z$. We have two cases: either (i) $z$ is a universal object (i.e. an object $z$ s.t. $\forall y(y \leq z)$ ), or (ii) not. If (i), then all $x x$ are parts of $z$, and hence $z \circ x x$. If (ii) then $z$ has a complement, $\bar{z}$. Hence, for each $x$ among $x x$ by supposition $\neg x \circ z$; thus $x \leq \bar{z}$. So $x x \leq \bar{z}$, which by the definition of $\mathrm{F}^{\prime \prime}$-type fusions implies that $a \leq \bar{z}$. By transitivity $z \leq \bar{z}$ which is impossible. Nota bene: this proof does not rely on antisymmetry.

${ }^{19}$ It is worth noting that this model — mutual parts with no other proper parts — is ruled out by QSP but not SSP. For those who feel the pull of the thought that 'if an object has a proper part, then it has two disjoint proper parts', one might consider adopting QSP in addition to SSP. Thanks to an anonymous referee on this point.
} 
Since $x_{2} \not \leq x_{1}$, by SSP we have it that there must be some part of $x_{2}$ that is disjoint from $x_{1}$. Likewise for any $x_{n}$ and $x_{n-1}$ which aren't mutual parts. So the relevant model becomes: ${ }^{20}$

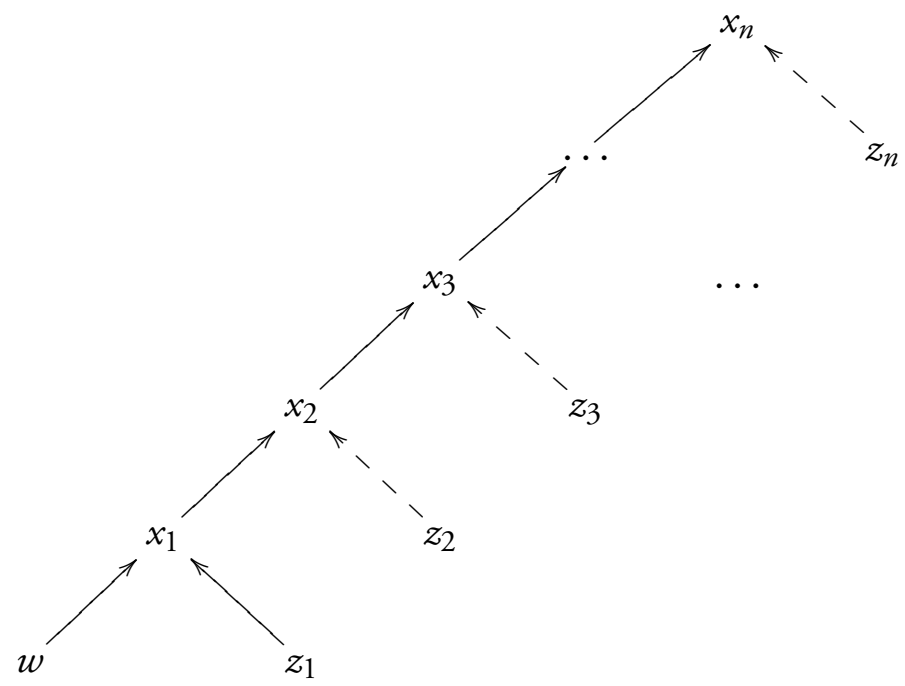

So, SSP and stronger supplementation principles can do the work that WSP was meant to do. Placing the blame for extensionality on supplementation is, I believe, a misdiagnosis of the problem. The real culprit is antisymmetry; denying it allows one a fully non-extensional mereology (in which all extensionality principles fail) ${ }^{21}$, without requiring us to accept strange models unrelated to anti-extensionalism.

What about the intuitive notion of proper parthood that WSP is supposed to capture? I suggest that WSP seems intuitive because we are not typically concerned with cases of coincident objects or non-extensionality. We typically run together two distinct notions of proper parthood: (i) the non-identical-part notion, and (ii) the part-with-remainder notion. In extensional cases, these two notions coincide. I have axiomatised the mutual parts mereology utilising notion (i). But the latter notion is also expressible in a mutual parts mereology: $x \leq y$ iff $x \leq y \wedge y \not \leq x$. This notion satisfies WSP (which follows immediately from the definition plus SSP). In previous work, I argued that $\lesseqgtr$ is the 'real' notion of proper parthood; however I now think that both

\footnotetext{
${ }^{20}$ This model is strongly supplemented. However, the model is incomplete, since it is missing some fusions (e.g. there is no fusion of $w$ and $z_{2}$ without $z_{1}$ ).

${ }^{21}$ For proofs, see $[6,8]$.
} 
notions are useful and that the difference is merely terminological. ${ }^{22}$ If 'proper parthood' means $<$, then WSP is false; if it means $\lesseqgtr$, then WSP is true.

The following axioms give a precise characterisation of the mutual parts approach.

Transitivity $(x<y \wedge y<z) \rightarrow x<z$

$\operatorname{SSSP} y \not \leq x \rightarrow \exists z \forall w(w \leq z \leftrightarrow(w \leq y \wedge \neg w \circ x))$

Unrestricted Fusion" $\forall x x \exists y \mathrm{~F}^{\prime \prime}(y, x x)$.

An equivalent axiomatization is given by:

Transitivity $(x<y \wedge y<z) \rightarrow x<z$

$\operatorname{SSP} y \not \leq x \rightarrow \exists z(z \leq y \wedge \neg z \circ x))$

Unrestricted Fusion' $\forall x x \exists y \mathrm{~F}^{\prime}(y, x x)$.

Moreover, since both approaches imply filtration, the mutual parts approach yields the existence of F-type fusions as well. As a result, the mutual parts approach is also compatible with Universalism of any sort, i.e. where 'fusion' here can be any of the $\mathrm{F}$-type, $\mathrm{F}^{\prime}$-type, or $\mathrm{F}^{\prime \prime}$-type definitions.

Universalism does not entail extensionalism after all. ${ }^{23}$

\footnotetext{
${ }^{22}$ See Rea [2 I] for more on this line of thought.

${ }^{23}$ Thanks to Ofra Magidor, Agustin Rayo, Achille Varzi, and the audience at the Necessity, Analyticity, \& the Apriori Workshop, University of Oslo (June 2013) for discussion of some of the issues in this paper. Thanks also to Micah Newman and two anonymous referees for comments on (earlier versions of) this paper.
} 


\section{References}

[i] Bennett, K. Spatio - temporal coincidence and the grounding problem. Philosophical Studies II8 (2004), 339-37I.

[2] Bohn, E. An argument against the necessity of unrestricted composition. Analysis 69, I (2009), 27-3I.

[3] Bunt, H. C. Mass Terms and Model-Theoretic Semantics. Cambridge University Press, Cambridge, 1985 .

[4] Caplan, B., Tillman, C., And Reeder, P. Parts of singletons. Journal of Philosophy io7 (2010), 50I-533.

[5] Carnap, R. Meaning and Necessity. University of Chicago Press, I947.

[6] Cotnoir, A. J. Anti-symmetry and non-extensional mereology. Philosophical Quarterly 60, 239 (2010), 396-405.

[7] Cotnoir, A. J. Strange parts: the metaphysics of non-classical mereology. Philosophy Compass 8,9 (2013), 834-845.

[8] Cotnoir, A. J., and Bacon, A. Non-wellfounded mereology. Review of Symbolic Logic 5, 2 (2OI 2), I 87-204.

[9] Donnelly, M. Using mereological principles to support metaphysics. Philosophical Quarterly 6I (20I I), 225-246.

[io] Forrest, P. Non-classical mereology and its application to sets. Notre Dame Journal of Formal Logic 43 (2002), 79-94.

[i i] Geach, P. On rigour in semantics. Mind 58, 232 (I949), 518-522.

[i 2] Gilmore, C. Quasi-supplementation, plenitudinous coincidentalism, and gunk. In Substance: New Essays. Philosophia Verlag, forthcoming.

[i3] Goodman, N. The Structure of Appearance. Harvard University Press, Cambridge, MA, I95I.

[i4] Hawthorne, J. Three Dimensionalism. Metaphysical Essays (2006), 8 5-I I I.

[ I 5] Hovda, P. What is classical mereology? Journal of Philosophical Logic 38, I (2009), 55-82.

[16] Kearns, S. Can a thing be part of itself? American Philosophical Quarterly 48, I (20 I I).

[i 7] Kleinschmidt, S. Multilocation and mereology. Philosophical Perspectives 25 (201 I), 253276.

[i 8] KosLicki, K. The Structure of Objects. Oxford University Press, 2008. 
[ig] Martin, R. Of time and the null individual. Journal of Philosophy 62 (I965), 723-736.

[20] McDaniel, K. Structure-making. Australasian Journal of Philosophy 87, 2 (2009), 25 I-274.

[2 I] Rea, M. Universalism and extensionalism: a reply to Varzi. Analysis 70, 3 (20 Iо), 490-496.

[22] Sanford, D. The problem of the many, many composition questions, and naïve mereology. Noûs 27, 2 (June 1993), 219-228.

[23] Simons, P. M. Parts: A Study In Ontology. Clarendon, Oxford, 987.

[24] Sмiтh, D. Mereology without weak supplementation. Australasian Journal of Philosophy 87 (2009), 505-5II.

[25] Thomson, J. J. Parthood and identity across time. Journal of Philosophy 80, 4 (April I983), $20 \mathrm{I}-220$.

[26] Thomson, J. J. The statue and the clay. Nồs 32, 2 (June I998), I 49-I73.

[27] Tillman, C., and Fowler, G. Propositions and parthood: The universe and antisymmetry. Australasian Journal of Philosophy 90 (2012), 525-539.

[28] Varzi, A. Mereology. In The Stanford Encyclopedia of Philosophy, E. N. Zalta, Ed. 2006.

[29] VArZI, A. A note on the transitivity of parthood. Applied Ontology I (2006), I 4 I-I 46.

[30] Varzi, A. The extensionality of parthood and composition. Philosophical Quarterly 58 (2008), I08-I33.

[3 I] VARZI, A. Universalism entails extensionalism. Analysis 69 (2009), 599-604. 